\title{
XIAP impairs mitochondrial function during apoptosis by regulating the Bcl-2 family in renal cell carcinoma
}

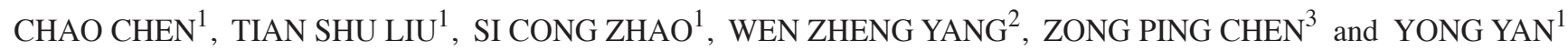 \\ Departments of ${ }^{1}$ Urology and ${ }^{2}$ Anesthesiology, Beijing Shijitan Hospital, Capital Medical University, \\ Beijing 100038; ${ }^{3}$ Department of Urology, The Affiliated Hospital of Zunyi Medical College, Zunyi 563000, P.R. China
}

Received July 22, 2017; Accepted March 13, 2018

DOI: $10.3892 /$ etm.2018.5974

\begin{abstract}
Efficient apoptosis requires Bcl-2 family-mediated mitochondrial outer membrane permeabilization (MOMP), which releases pro-apoptotic proteins to the cytosol, activating apoptosis and inhibiting X-linked inhibitor of apoptosis protein (XIAP). XIAP is a member of the inhibitors of apoptosis protein family whose expression is elevated in many cancer types and participates in the release of pro-apoptotic proteins. To explore the association between XIAP and the Bcl-2 family, and the influence of XIAP on mitochondria, RNA interference of XIAP was performed in Caki-1 cells and the dynamic change in the levels of related proteins was compared with the original Caki-1 cells upon induction of apoptosis. Upon knockdown of XIAP, the release of cytochrome $c$ (Cyt-c), second mitochondria-derived activator of caspase (Smac) and apoptotic protease activating factor 1 (Apaf-1) from mitochondria proceeded normally, whereas in Caki-1 cells, the release of these pro-apoptotic proteins was significantly prolonged, and incomplete. Downregulation of XIAP through small interfering RNA resulted in an increase of apoptosis and a marked decrease in Bcl-2 and Bcl-xl levels at $3 \mathrm{~h}$. Additionally, the regulation of the level of XIAP protein affected the specific ratios of $\mathrm{Bcl}-2 / \mathrm{Bax}$ and $\mathrm{Bcl}-\mathrm{xl} / \mathrm{Bax}$, which play decisive roles in cell death. In the present study, it was revealed that XIAP can feed back to mitochondria, delaying Cyt-c and Apaf-1 release. Furthermore, XIAP can limit the release of its inhibitor Smac with the involvement of Bcl-2 family proteins.
\end{abstract}

\section{Introduction}

Apoptosis, which is the best-described form of programmed cell death, is a controlled and energy-dependent process, the

Correspondence to: Professor Yong Yan, Department of Urology, Beijing Shijitan Hospital, Capital Medical University, 10 Iron Medical Road, Beijing 100038, P.R. China

E-mail: yanyongcq@163.com

Key words: XIAP, Bcl-2 family proteins, apoptosis, renal cell carcinoma deregulation of which can lead to cancer (1). Over the past two decades, we have witnessed explosive progress in this field. It is now known that there are at least 3 pathways in apoptosis: The endogenous mitochondrial pathway, the stress endoplasmic reticulum pathway and the exogenous death receptor pathway (2). Among these, the role of the mitochondrial pathway had been neglected for a long time. However, researchers have found that mitochondria play an important role in apoptosis by releasing key effector proteins from the mitochondrial intermembrane space (IMS), including cytochrome $c(\mathrm{Cyt}-\mathrm{c})$ and second mitochondria-derived activator of caspase (Smac)/DIABLO. Mitochondrial outer membrane permeabilization (MOMP) results in the release of IMS proteins, which supports the activation of executioner caspases and is paramount for effective killing of cancer cells $(3,4)$.

Research has shown that the MOMP is the most important point in apoptosis and that the mitochondrial pathway of apoptosis is characterized by MOMP and activation of Cyt-c (5). Moreover, the interaction between the members of the Bcl-2 family can control the MOMP (6). Bcl-2 family proteins are subdivided into two groups on the basis of their pro- or anti-apoptotic action. Anti-apoptotic family members include Bcl-2, Bcl-xl, Bcl-w, Mcl-1 and A1/Bfl-1, while pro-apoptotic family members include Bax, Bak and Bok/Mtd (7,8). Bcl-2 and Bcl-xl play anti-apoptotic roles by inhibiting their pro-apoptotic counterparts and blocking the activation of caspase in cytoplasm. The multidomain pro-apoptotic proteins, Bax and Bak, are responsible for MOMP and are the master effectors of apoptosis (9). In some cell types, the extrinsic pathway can also cross with the mitochondrial pathway through caspase- 8 mediated cleavage of Bid, and the truncated $\mathrm{tBid}$ will translocate to the mitochondria to trigger Cyt-c release (10).

The expression and regulation of the Bcl-2 family has a significant influence on the apoptosis. X-linked inhibitor of apoptosis protein (XIAP) is the most potent caspase inhibitor of the IAP family, whose inhibitory effects in the mitochondria during apoptosis are well documented. In addition to its well-known function in caspase suppression, XIAP can permeabilize and enter mitochondria (11). XIAP comprises three baculoviral IAP repeat domains (BIR1-3) and an interesting zinc-finger RING domain, which exerts E3 ubiquitin ligase activity (12). The XIAP E3 ligase activity recruits endolysosomes into mitochondria, resulting in Smac 
degradation. Through its E3 ligase, XIAP may possess more potential biological functions.

So far, the mechanism by which XIAP regulates mitochondrial function is not clear. Previous study has demonstrated that XIAP serves as an E3 ligase for Bcl-2 and stimulates UPS (Ubiquitin Proteasome System)-mediated degradation of Bcl-2 (13). Since, there is a close relationship between the Bcl-2 family and mitochondrial function, we put forward the hypothesis that XIAP may impact mitochondrial function during apoptosis by regulating the Bcl-2 family. We also investigated whether physiological or elevated XIAP levels have a comprehensive effect on mitochondria.

Levels of XIAP were shown to be elevated in renal cell carcinomas compared with normal renal cells. Previous research has found that renal cell carcinomas are resistant to apoptosis induced by chemical, immunological preparations and radiotherapy. Moreover, a poor prognosis is associated with the over-expression of XIAP in renal cell carcinomas (14). Therefore, we chose the Caki-1 cell line, which over-express XIAP, and established a stable transfected Caki-1 cell line with knockdown of XIAP using RNA interference. Using these cells, we studied the dynamic change of the related proteins in the process of apoptosis between the two cell lines.

\section{Materials and methods}

Plasmids and molecular cloning. The XIAP sequence targeted by RNA interference in this study was based on the data of Bilim et al (15), which verified that there were no homologous sequences between this fragment and other gene sequences and that the shRNA contained in this fragment can effectively knockdown expression of the XIAP protein. The target sequence is 5'-AGGTGAAGGTGATAAAGTA-3'. The BLOCK-iT ${ }^{\mathrm{TM}}$ U6 RNAi Entry Vector kit (Invitrogen; Thermo Fisher Scientific, Inc., Waltham, MA, USA) was used to generate the RNAi construct. Based on the selected target sequence, the following oligonucleotide sequences for the shRNA were used 5'-CACCGAGGTGAAGGTGATAAA GTACGAATACTTTATCACCTTCACC-3' (top strand DNA oligo); 5'-AAAAGGTGAAGGTGATAAAGTATTCGTACT TTATCACCTTCACCTC-3' (bottom strand DNA oligo).

These siRNA-encoding complementary single-stranded oligonucleotides were hybridized to give Xho I-and Hind III-compatible overhangs and then were ligated into $\mathrm{pENTR}^{\mathrm{TM}} / \mathrm{U} 6$. The constructed plasmids were confirmed by sequencing and named XIAP-shRNA-pENTR ${ }^{\mathrm{TM}} / \mathrm{U} 6$.

Cell culture and transfection. Caki-1 cells (renal carcinoma cell with over-expression of XIAP) were purchased from China Infrastructure of Cell Line Resources (Beijing, China). The cells were cultured in minimum essential medium (MEM; Gibco; Thermo Fisher Scientific, Inc.) supplemented with 10\% FBS (Tianjin Kang Yuan Biological Technology Co., Ltd., Tianjin, China). The cells were maintained in a humidified $37^{\circ} \mathrm{C}$ incubator with $5 \% \mathrm{CO}_{2}$. The cells were split twice weekly. Cells in the logarithmic growth phase were used for experiments.

Lipofectamine 2000 transfection reagent was used for cell transfection (Gibco; Thermo Fisher Scientific, Inc.). The BLOCK-iT ${ }^{\mathrm{Tm}}$ U6 RNAi Entry Vector kit (Tianjin Kang
Yuan Biological Technology Co., Ltd.) was used to generate a construct to knock down XIAP expression. Transfected cells were selected with G418 to generate stable transfected clonal cells (Caki-1 cells successfully transfected had G418 resistance) for 3-4 weeks. Stably transfected Caki-1 cell clones were collected and named Caki-1/XIAP-shRNA-pENTR ${ }^{\mathrm{TM}}$. with Caki-1/pENTR ${ }^{\mathrm{TM}}$ cells used as controls. Because the PENTR $^{\mathrm{TM}} / \mathrm{U} 6$ plasmid carries the GFP gene we used a fluorescence microscope (AMG; Thermo Fisher Scientific, Inc.) to identify transfected and no-transfected cells. After the formation of the clonal cell lines, we picked a monoclonal cell colony with green fluorescence and transferred it to a 24-well plate for amplification.

Measurement of cell viability. Cell viability was determined by the MTT assay using 3-(4,5-dimethylthiazol-2-yl)-2,5-diphenyltetrazolium bromide. We used a MTT-kit (Gibco; Thermo Fisher Scientific, Inc.) to detect the inhibition rate of cells exposed to different drug concentrations. Etoposide was used to induce apoptosis.

Preparation of cell lysate and western blotting. Total protein was extracted at different time points after the induction of apoptosis. The extraction of cytoplasmic and mitochondrial proteins was completed according to the Mitochondrial Isolation kit instructions. Protein concentration was determined using a bicinchoninic acid (BCA) protein assay kit. Whole cell lysates were prepared using the RIPA reagent (Bioeasytech, Beijing, China). A total of $40 \mu \mathrm{g}$ of protein from each sample was resolved by SDS-polyacrylamide gel electrophoresis (PAGE) and transferred to polyvinylidene fluoride (PVDF) membranes. The membranes were blocked with 5\% non-fat milk overnight and probed with primary antibodies against target proteins at $4^{\circ} \mathrm{C}$ overnight, followed by incubation with a secondary anti-rabbit IgG-HRP antibody (1:10,000 dilution; Bioeasytech) or anti-mouse IgG-HRP antibody (1:20,000 dilution; Bioeasytech) at $37^{\circ} \mathrm{C}$ for $45 \mathrm{~min}$. The following antibodies were used: anti-XIAP, anti-apoptotic protease activating factor 1 (Apaf-1), anti-Smac, anti-Bcl-2, anti-Bax, anti-Bcl-xl (diluted 1:1,000; Cell Signaling Technology, Inc., Danvers, MA, USA), anti-Cyt-c (diluted 1:500; Cell Signaling Technology, Inc.). Target bands were visualized by enhanced chemiluminescence (ECL) solution (WBKLS0500; EMD Millipore, Billerica, MA, USA) and analysed by Gel-Pro-Analyzer software (Media. Cybernetics, Inc., Bethesda, MD, USA). GAPDH was served as an internal control for cytoplasmic samples, and COX IV was used as a loading control for mitochondrial samples.

Statistical analysis. All values are reported as the mean \pm standard deviation, and differences between groups were analysed using a two-tailed t-test or one-way analysis of variance (ANOVA) with the Student-Newman-Keuls test, Statistical analysis was performed with SPSS 17.0 software (SPSS, Inc., Chicago, IL, USA).

\section{Results}

Effect of siRNA on protein expression of XIAP gene. To reduce the expression of XIAP in Caki-1 cells, sequence-specific siRNA of XIAP was synthesized, as mentioned in the 


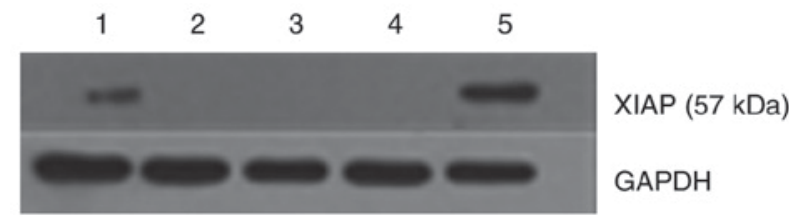

Figure 1. Western blotting for X-linked inhibitor of apoptosis protein (XIAP). 1-3, represent three different lines of cells transfected with the shRNA-pENTR ${ }^{\mathrm{TM}} / \mathrm{U} 6-\mathrm{XIAP}$ group. Lane 1 represent empty plasmid group; lanes 2-4 represent cell lines with knock-down XIAP; lane 5 represent original Caki-1 cells. By means of grey scale measurement, the relative content of XIAP expression was expressed as XIAP/GAPDH. XIAP expression in cells expressing $\mathrm{pENTR}^{\mathrm{TM}} / \mathrm{U} 6$ was $23.6 \%$; in the three cell lines expressing shRNA-pENTR ${ }^{\mathrm{TM}} / \mathrm{U} 6-\mathrm{XIAP}$, it was $0 \%$; and the original Caki-1 cells (which overexpress XIAP), it was $64.5 \%$.

Materials and methods section. The transfected cells were selected with for 3-4 weeks to generate stable transfected clones. Three stable transfected cell clones were screened and one was selected for further experiments. The interference efficiency of XIAP knockdown in cells was confirmed by western blot. As shown in Fig. 1, XIAP shRNA transfection markedly reduced the expression of XIAP, while there was no significant difference between the Caki-1 control group and the $\mathrm{pENTR}^{\mathrm{TM}} / \mathrm{U} 6$ group.

Knockdown of XIAP-sensitized Caki-1 cells to etoposide. To evaluate whether the down-regulation of XIAP expression would influence the anti-apoptotic ability of Caki-1 cells, the MTT method was used to detect the cell death rate under different drug concentrations. As shown in Fig. 2, we observed that after knockdown of XIAP protein expression, cell death rate was significantly increased in response to different drug concentrations, while there was no statistically significant difference between original Caki-1 cells and pENTR $^{\mathrm{TM}} \mathrm{U} 6$ transfected Caki-1 cells.

XIAP impairs the release of pro-apoptotic proteins from mitochondria. To comprehensively understand the effect of XIAP on mitochondria, cells (Caki-1 and shRNA-XIAP-1) were treated with etoposide for the indicated time intervals. At the end of etoposide treatment, cytosolic and mitochondrial fractions were isolated, and equal amounts of protein were subjected to western blotting to detect Cyt-c, Smac, and Apaf-1). As shown in Fig. 3, comparing the dynamic changes of proteins in the two cell lines, we found that the levels of mitochondrial Cyt-c, Smac and Apaf-1 did not decrease rapidly in response to etoposide in Caki-1 cells. Prior to $24 \mathrm{~h}$, we did not detect an obvious reduction in protein levels, indicating that the release of pro-apoptotic proteins from mitochondria was delayed or inhibited in response to the overexpression of XIAP in these renal carcinoma cells. In contrast, in shRNA-XIAP-1-expressing cells (knockdown of XIAP), the levels of mitochondrial Cyt-c, Smac and Apaf-1 decreased rapidly after etoposide stimulation and by $6 \mathrm{~h}$ after stimulation we could hardly detect these three proteins in the mitochondria, meaning that after knockdown of XIAP in Caki-1 cells, the release time of pro-apoptotic proteins from mitochondria was decreased relative to cells overexpressing XIAP. Additionally, we found that Apaf-1 expression
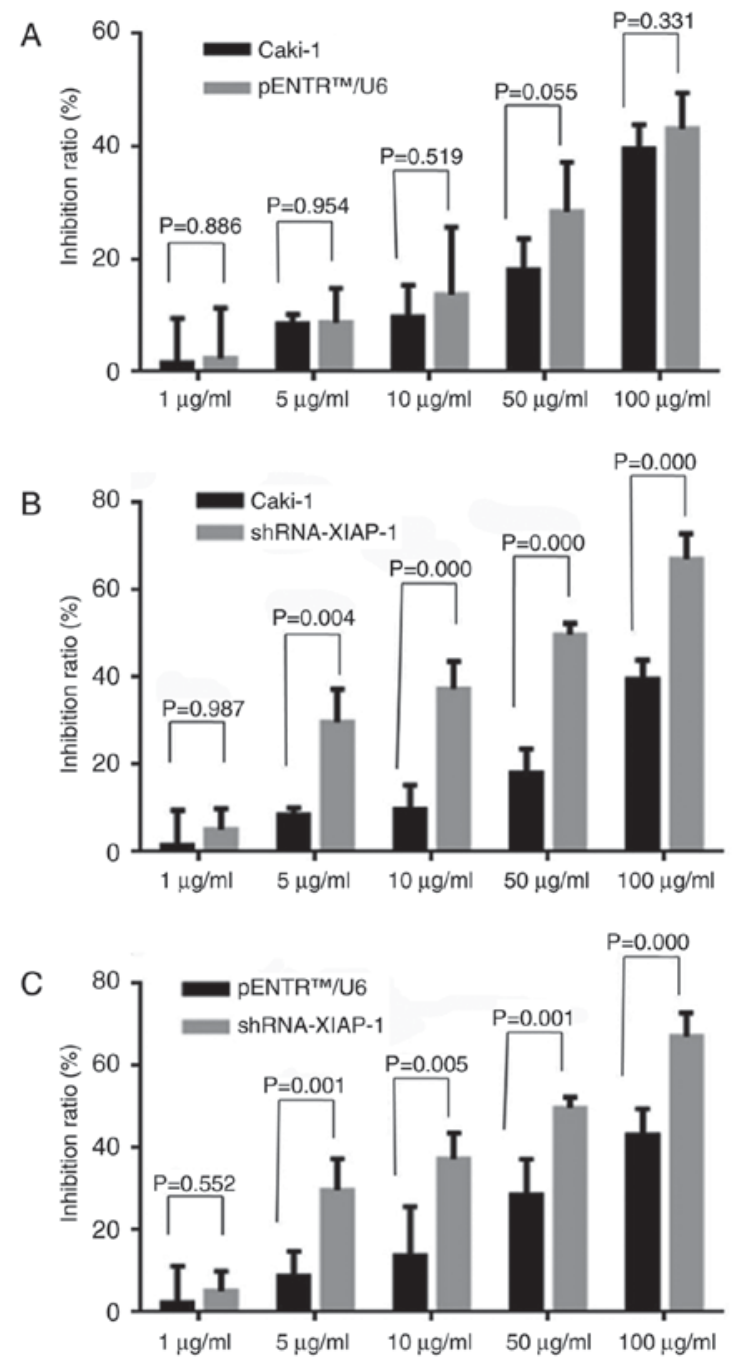

Figure 2. Cell inhibition rate of different groups. (A) Cell inhibition rate of Caki-1 and pENTR ${ }^{\mathrm{TM}} \mathrm{U} 6$ groups. The inhibitory rate of the cells was not significantly different between the two groups. However, in the (B) Caki-1 and shRNA-X-linked inhibitor of apoptosis protein (XIAP)-1 groups, except for the $1-\mu \mathrm{g} / \mathrm{ml}$ concentration, there were significant differences between concentrations. (C) Comparing $\mathrm{pENTR}^{\mathrm{TM}} / \mathrm{U} 6$ with shRNA-XIAP-1, the results showed a significant difference between the two groups.

was decreased at $0.5 \mathrm{~h}$, which was more rapid than for the other proteins. We speculate that the release mechanism of Apaf- 1 is different from the other proteins. The above results indicate that overexpression of XIAP impairs the release of pro-apoptotic proteins from mitochondria and thereby inhibits apoptosis. During apoptosis, Smac, which is similar to Cyt-c, is released into the cytosol, and all pro-apoptotic proteins play similar roles in the cytoplasm.

Therefore, we further confirmed the effect of XIAP on mitochondria by detecting the dynamic changes of these proteins in the cytoplasm. As shown in Fig. 4 and consistent with previous results, Cyt-c and Smac were not detected in the cytoplasm until $12 \mathrm{~h}$ after apoptosis stimulation, while Apaf-1 was detected in cytoplasm at $3 \mathrm{~h}$ after stimulation. Upon knockdown of XIAP there was a noticeable difference with pro-apoptotic proteins more rapidly increase after the stimulus, with all three pro-apoptotic proteins being detected in cytoplasm at $0.5 \mathrm{~h}$. The time point of protein release from mitochondria was obviously earlier than in cells 

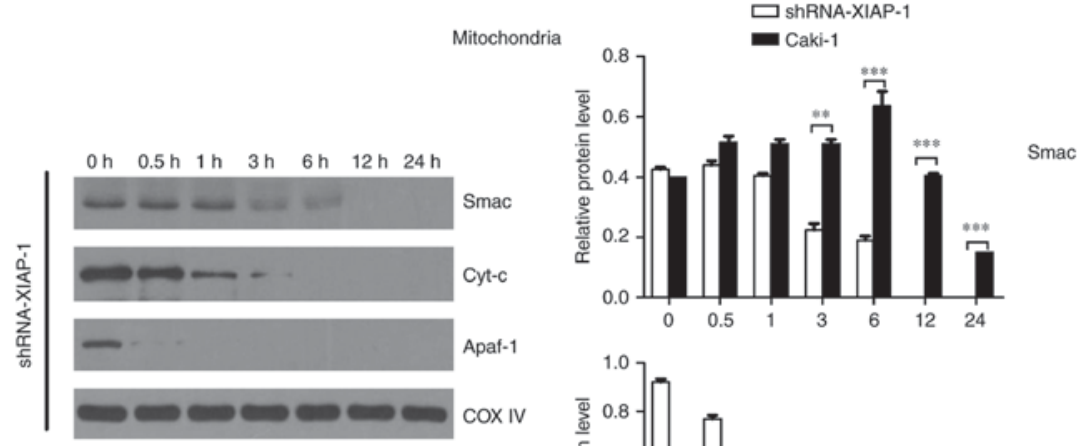

$0 \mathrm{~h} \quad 0.5 \mathrm{~h} 1 \mathrm{~h} \quad 3 \mathrm{~h} \quad 6 \mathrm{~h} \quad 12 \mathrm{~h} \quad 24 \mathrm{~h}$
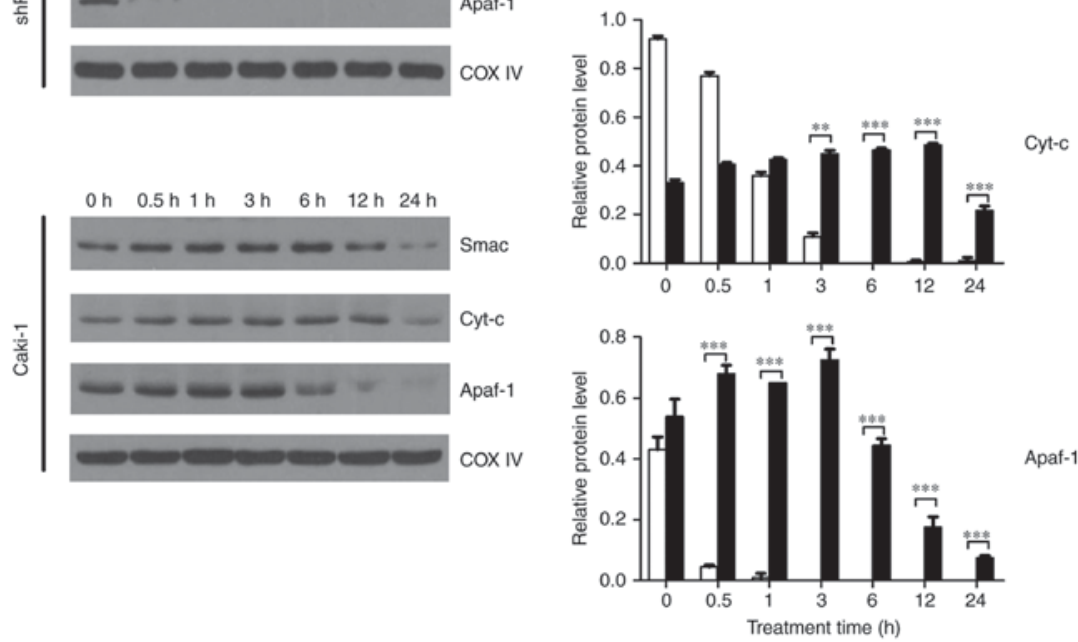

Figure 3. Immunoblotting for pro-apoptotic proteins inside the mitochondria. Caki-1 cells and siRNA transfected Caki-1 [shRNA-X-linked inhibitor of apoptosis protein (XIAP)-1] cells were treated with etoposide for the indicated times and western blotting was used to detect the mitochondrial second mitochondria-derived activator of caspase (Smac), cytochrome $c$ (Cyt-c) and apoptotic protease activating factor 1 (Apaf-1) protein levels. COX IV was used as a loading control. The relative protein levels of Smac, Cyt-c and Apaf-1 were normalized to COX IV expression as determined by densitometry. Data represent the mean values $\pm \operatorname{SEM}(\mathrm{n}=3),{ }^{* *} \mathrm{P}<0.01,{ }^{* * *} \mathrm{P}<0.001$.
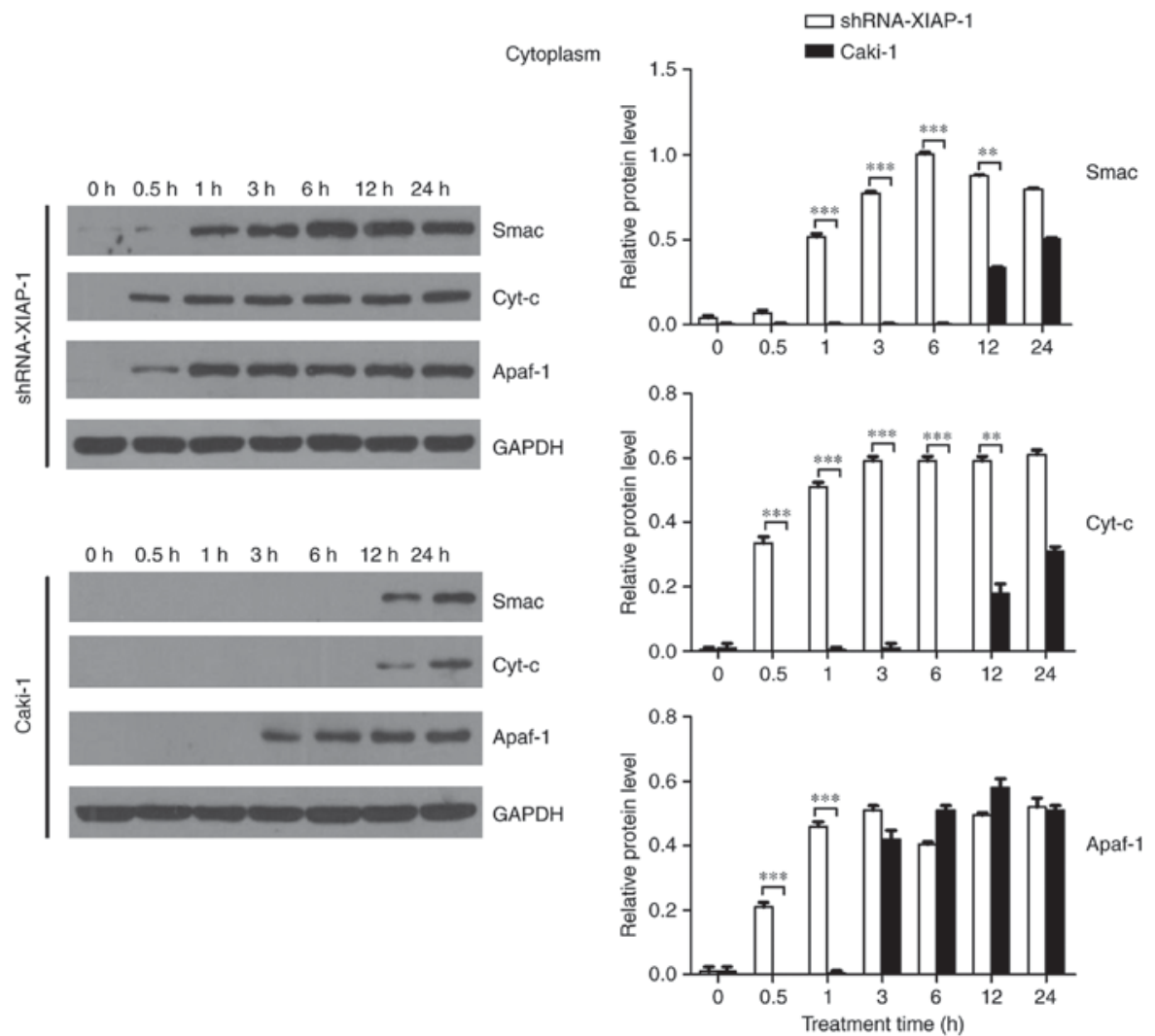

Figure 4. Immunoblotting for pro-apoptotic proteins in the cytoplasm. Western blotting validation of intracytoplasmic second mitochondria-derived activator of caspase (Smac), cytochrome $c$ (Cyt-c) and apoptotic protease activating factor 1 (Apaf-1) expression in Caki-1 cells vs. X-linked inhibitor of apoptosis protein (XIAP)-knockdown cells. GADPH was used as a loading control. The relative protein levels of pro-apoptotic proteins were normalized to GAPDH expression as determined by densitometry. Data represent the mean values $\pm \operatorname{SEM}(n=3),{ }^{* *} \mathrm{P}<0.01,{ }^{* * *} \mathrm{P}<0.001$. 

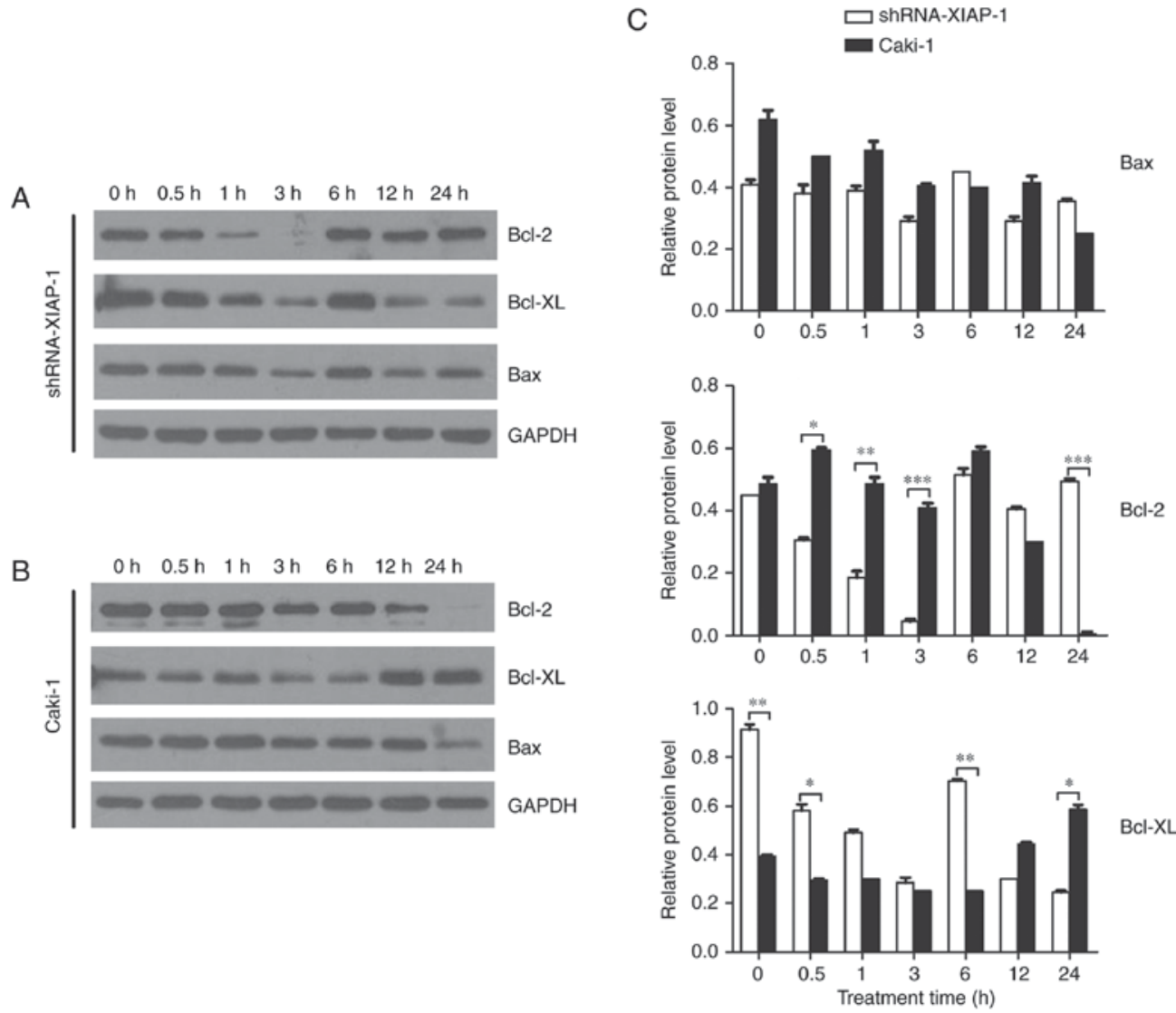

Figure 5. Immunoblotting for Bcl-2 family proteins in the cytoplasm. Western blotting validation of intracytoplasmic Bax, Bxl-2 and Bcl-xl expression in (A) X-linked inhibitor of apoptosis protein (XIAP)-knockdown cells and (B) Caki-1 cells. GADPH was used as a loading control. (C) Relative expression of Bcl-2 family proteins after etoposide treatment were shown in the right part. Values are means \pm standard deviation of 3 experiments. ${ }^{*} \mathrm{P}<0.05$, ${ }^{* *} \mathrm{P}<0.01$, ${ }^{* * * *} \mathrm{P}<0.001$

overexpressing XIAP, supporting the hypothesis that XIAP impairs the transfer of proteins from the mitochondria to the cytoplasm. Moreover, the change in Smac translocation was similar to that of Cyt-c. However, the trend for Apaf-1 was different to that of Smac and Cyt-c. Thus, XIAP may affect the release of Apaf-1 through a different mechanism.

Bcl-2 family protein expression is related to the XIAP protein. A number of studies have shown that the $\mathrm{Bcl}-2$ family plays an important role in the mitochondrial apoptosis pathway and that the major site of action of the Bcl-2 family of proteins is on the mitochondrial membrane. Therefore, we studied dynamic changes in the Bcl-2 family proteins after the stimulation of apoptosis, observing if there were differences between cell lines overexpressing XIAP and cell lines with XIAP expression knocked down. We selected Bcl-2 and $\mathrm{Bcl}-\mathrm{xl}$ as our target pro-survival proteins and Bax as our target pro-apoptotic protein. From the results (Fig. 5), we can see that after the stimulation of apoptosis, the pro-survival proteins, Bcl-2 and Bcl-xl, were not significantly changed in Caki-1 cells. However, in cells with XIAP expression knocked down, the level of Bcl-2 and Bcl-xl decreased 10 -fold and 4-fold, respectively at $3 \mathrm{~h}$ after etoposide treatment, which indicates a decrease in the restrainting effects upon Bax and Bak. For the pro-apoptotic proteins, we found that there was no significant difference in Bax expression in these two cell lines at each time point, indicating that the
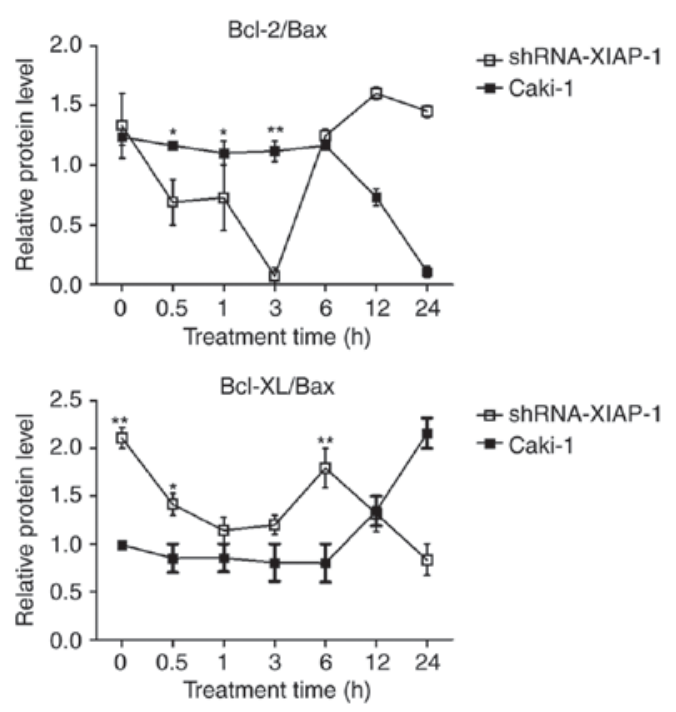

Figure 6. Expression ratios Bcl-2/Bax and Bcl-xl/Bax. Fold-change of relative expression of the ratios of $\mathrm{Bcl}-2 / \mathrm{Bax}$ and $\mathrm{Bcl}-\mathrm{xl} / \mathrm{Bax}$, as detected by western blotting. The balance of Bcl-2/Bax and Bcl-xl/Bax was not changed until $12 \mathrm{~h}$ in Caki-1 cells. However, the balance was immediately broken after the stimulation of apoptosis in X-linked inhibitor of apoptosis protein (XIAP)-knockdown cells. Values are means \pm standard deviation of 3 experiments. ${ }^{*} \mathrm{P}<0.05,{ }^{* *} \mathrm{P}<0.01$.

function of Bax/Bak may only be regulated by a series of conformational changes. 
Imbalance of Bcl-2 family proteins. Studies suggest that the mitochondrial apoptosis pathway is regulated by balanced interactions between members of the Bcl-2 family of proteins (16). Therefore, we analysed the Bcl-2/Bax and Bcl-xl/Bax ratios (Fig. 6). In Caki-1 cells, the balance of $\mathrm{Bcl}-2 / \mathrm{Bax}$ and $\mathrm{Bcl}-\mathrm{xl} / \mathrm{Bax}$ were impaired at $12 \mathrm{~h}$ in accordance with the time of release of pro-apoptotic proteins from mitochondria, which was extended compared to the shRNA-XIAP-1 expressing cells. After knockdown of XIAP, the ratio was impaired at $0.5 \mathrm{~h}$ after the stimulation of apoptosis, which was also in accordance with the time of release of pro-apoptotic proteins from mitochondria in shRNA-XIAP-1 expressing cells. From these results, we can see that the change in XIAP expression impacts the balance of Bcl-2 family proteins, which shows that XIAP has an important impact upon the Bcl-2 family of proteins.

\section{Discussion}

Mitochondria play an important role in the process of apoptosis, with Bcl-2 family proteins and Cyt-c closely interacting with each other in the mitochondrial apoptotic pathway (17). However, key issues regarding upstream regulation of the Bcl-2 family remain largely unclear. In addition to its well-known role in caspase suppression, XIAP can localize to the mitochondria and activate Bax-mediated mitochondrial permeabilization. Moreover, we previously reported that XIAP can enter $\mathrm{BH} 3$-only protein-targeted or CCCP-depolarized mitochondria, resulting in the degradation of its antagonist, Smac, through endolysosomal and proteasomal actions (18). As MOMP-mediated release of Smac and the consequent inhibition of XIAP are essential for activation of apoptosis (19), this suggests an additional pro-survival role for XIAP during apoptosis. Therefore, we first studied the impact of XIAP on mitochondria. We then observed changes in the Bcl-2 family of proteins to determine the function and regulation of the $\mathrm{Bcl}-2$ family by XIAP during apoptosis signalling by regulating the level of XIAP through RNA small interference.

The Bcl-2 family is a class of very conserved proteins. Bcl-2 and Bcl-xL play a major role in the outer membrane of the mitochondria by maintaining the integrity of the membrane. They are the major anti-apoptotic proteins. Bax and Bak can destroy the integrity of mitochondrial membrane, promoting the release of Cyt-c and mediating apoptosis. They are the major pro-apoptotic proteins (20). Therefore, we selected Bcl-2, Bcl-xl and Bax as our targets to detect the effects of altered XIAP expression.

Apoptosis is a rapid process. Therefore, it is very meaningful to study the dynamics of protein-protein and protein-membrane interactions in vivo as well as the structures of membrane-bound proteins and captured conformational changes, which are the key to understanding the mechanisms of both apoptosis induction and execution. Therefore, in order to comprehensively and dynamically evaluate the function of the Bcl-2 family proteins and mitochondria, we studied the dynamic changes in pro-apoptotic proteins between the cytoplasmic and mitochondrial compartments at different time points (at $0,0.5,1,3,6,12$ and $24 \mathrm{~h}$ ) before and after the stimulation of apoptosis.

We showed that, compared with the knockdown of XIAP, the presence of XIAP can prolong and limit the release of Cyt-c, Smac and Apaf-1 from the mitochondria during etoposide-induced apoptosis. The major goal of the mitochondrial apoptosis pathway is the release of caspase activator (e.g., Cyt-c). The release of Cyt-c occurs following a variety of death stimuli and has been shown to activate Apaf-1, which in turn activates caspase-9 and caspase-3 (21). The release of these pro-apoptotic proteins is a marker of the mitochondrial apoptosis pathway. Thus, the results of our studies clearly show that XIAP affects the function of the mitochondria during apoptosis. Additionally, we found that the changes in expression of Apaf-1 are different from that of the other proteins, which indicates that the release mechanism of Apaf- 1 is different. This difference in release mechanism of these proteins needs to be studied more deeply. Another question is how XIAP affect mitochondria. Thomas et al evaluated the apoptotic response to drug treatment of B-CLL cells in vitro, finding that cells with a high $\mathrm{Bcl}-2 / \mathrm{Bax}$ ratio were more drug resistant than cells with a low $\mathrm{Bcl}-2 / \mathrm{Bax}$ ratio (22). In addition, researchers have shown that the $\mathrm{Bcl}-2 / \mathrm{Bax}$ ratio correlates with ID50 values and clinical responsiveness (23). Comparing the ratio of Bcl-2/Bax and $\mathrm{Bcl}-\mathrm{xl} / \mathrm{Bax}$ in the two cell lines, our data indicate that the level of Bcl-2 family members is primarily mediated by XIAP and the balance of Bcl-2 family proteins is related to XIAP. Thus, overexpression of XIAP influences changes in Bcl-2 family members during the process of cell apoptosis, which affect the function of mitochondria.

Taken together, our data show that the activity of XIAP induced a previously unknown function at the mitochondria and in the Bcl-2 family proteins. This study strengthens the anti-apoptotic potential of XIAP and provides a new direction for the treatment of cancer.

\section{Acknowledgements}

This study was supported by grants from National Natural Science Foundation of China (no. 81441073) and Beijing Municipal Commission of education science and technology plan projects (no. KM201310025017).

\section{References}

1. Burz C, Berindan-Neagoe I, Balacescu O and Irimie A: Apoptosis in cancer: Key molecular signaling pathways and therapy targets. Acta Oncologica 48: 811-821, 2009.

2. Jeong SY and Seol DW: The role of mitochondria in apoptosis BMB Rep 41: 11-22, 2008.

3. Newmeyer DD and Fergusonmiller S: Mitochondria: Releasing power for life and unleashing the machineries of death. Cell 112: 481-490, 2003.

4. Vo TT: Relative mitochondrial priming of myeloblasts and normal HSCs determines chemotherapeutic success in AML. Cell 151: 344-355, 2012.

5. Oltvai ZN, Milliman CL and Korsmeyer SJ: Bcl-2 heterodimerizes in vivo with a conserved homolog, Bax, that accelerates programmed cell death. Cell 74: 609-619, 1993.

6. Chipuk JE and Green DR: How do BCL-2 proteins induce mitochondrial outer membrane permeabilization? Trends Cell Biol 18: 157-164, 2008.

7. Youle RJ and Strasser A: The BCL-2 protein family: Opposing activities that mediate cell death. Nat Rev Mol Cell Biol 9: 47-59, 2008.

8. Kvansakul M, Yang H, Fairlie WD, Czabotar PE, Fischer SF, Perugini MA, Huang DC and Colman PM: Vaccinia virus anti-apoptotic F1L is a novel Bcl-2-like domain-swapped dimer that binds a highly selective subset of $\mathrm{BH} 3$-containing death ligands. Cell Death Differ 15: 1564-1571, 2008. 
9. Wei MC, Zong WX, Cheng EH, Lindsten T, Panoutsakopoulou V, Ross AJ, Roth KA, Macgregor GR, Thompson CB and Korsmeyer SJ: Proapoptotic BAX and BAK: A requisite gateway to mitochondrial dysfunction and death. Science 292: 727-730, 2001 .

10. Li H, Zhu H, Xu CJ and Yuan J: Cleavage of BID by caspase 8 mediates the mitochondrial damage in the Fas pathway of apoptosis. Cell 94: 491-501, 1998

11. Hamacher-Brady A and Brady NR: Bax/Bak-dependent, Drpl-independent Targeting of X-linked inhibitor of apoptosis protein XIAP) into inner mitochondrial compartments counteracts smac/DIABLO-dependent effector caspase activation. J Biol Chem 290: 22005, 2015

12. Shiozaki EN, Chai J, Rigotti DJ, Riedl SJ, Li P, Srinivasula SM, Alnemri ES, Fairman R and Shi Y: Mechanism of XIAP-mediated inhibition of caspase-9. Mol Cell 11: 519-527, 2003.

13. Edison N, Curtz Y, Paland N, Mamriev D, Chorubczyk N, Haviv-Reingewertz T, Kfir N, Morgenstern D, Kupervaser M, Kagan J, et al: Degradation of Bcl-2 by XIAP and ARTS promotes apoptosis. Cell Rep 21: 442-454, 2017.

14. Yan Y, Mahotka C, Heikaus S, Shibata T, Wethkamp N, Liebmann J, Suschek CV, Guo Y, Gabbert HE, Gerharz CD and Ramp U: Disturbed balance of expression between XIAP and Smac/DIABLO during tumour progression in renal cell carcinomas. Br J Cancer 91: 1349-1357, 2004.

15. Bilim V, Yuuki K, Itoi T, Muto A, Kato T, Nagaoka A, Motoyama T and Tomita Y: Double inhibition of XIAP and Bcl-2 axis is beneficial for retrieving sensitivity of renal cell cancer to apoptosis. Br J Cancer 98: 941-949, 2008

16. Gavathiotis E, Reyna DE, Davis ML, Bird GH and Walensky LD: $\mathrm{BH} 3$-triggered structural reorganization drives the activation of proapoptotic BAX. Mol Cell 40: 481-492, 2010.
17. Tait SW and Green DR: Mitochondria and cell death: Outer membrane permeabilization and beyond. Nat Rev Mol Cell Biol 11: 621-632, 2010.

18. Hamacherbrady A, Choe SC, Krijnselocker J and Brady NR: Intramitochondrial recruitment of endolysosomes mediates Smac degradation and constitutes a novel intrinsic apoptosis antagonizing function of XIAP E3 ligase. Cell Death Differ 21: 1862-1876, 2014.

19. Jost PJ, Grabow S, Gray D, Mckenzie MD, Nachbur U, Huang DC, Bouillet P, Thomas HE, Borner C, Silke J, et al: XIAP discriminates between type I and type II FAS-induced apoptosis. Nature 460: 1035-1039, 2009.

20. Adams JM and Cory S: The Bcl-2 apoptotic switch in cancer development and therapy. Oncogene 26: 1324-1337, 2007.

21. Bossy-Wetzel E, Newmeyer DD and Green DR: Mitochondrial cytochrome $c$ release in apoptosis occurs upstream of DEVD-specific caspase activation and independently of mitochondrial transmembrane depolarization. EMBO J 17: 37-49, 1998.

22. Thomas A, El Rouby S, Reed JC, Krajewski S, Silber R, Potmesil M and Newcomb EW: Drug-induced apoptosis in B-cell chronic lymphocytic leukemia: Relationship between p53 gene mutation and bcl-2/bax proteins in drug resistance. Oncogene 12: 1055-1062, 1996.

23. Pepper C, Hoy $\mathrm{T}$ and Bentley DP: $\mathrm{Bcl}-2 / \mathrm{Bax}$ ratios in chronic lymphocytic leukaemia and their correlation with in vitro apoptosis and clinical resistance. $\mathrm{Br} \mathrm{J}$ Cancer 76 : 935-938, 1997. 